\title{
Cardiac mechanics, calcium overload and arrhythmogenesis
}

\author{
Kursanov A. ${ }^{1 *}$, Solovyova O. ${ }^{1,2}$, Katsnelson L. ${ }^{1}$, Medvedev K. ${ }^{2}$, Vasilyeva A. ${ }^{1,2}$, Vikulova N. ${ }^{1}$, \\ Markhasin V.S. ${ }^{1,2}$ \\ ${ }^{1}$ Institute of Immunology and Physiology UB of RAS, Yekaterinburg, Russia \\ ${ }^{2}$ Ural Federal University, Yekaterinburg, Russia \\ *e-mail: alexander.kursanov@gmail.com
}

Key words: calcium overload, rhythm disturbances, cardiac mechanics

Motivation and Aim: It is well-known that $\mathrm{Ca}^{2+}$ overload may cause cardiac arrhythmia. However, possible contribution of the mechanical factors to the arrhythmia development in $\mathrm{Ca}^{2+}$-overloaded cardiomyocytes has been insufficiently addressed. Earlier we have developed a mathematical model of cardiomyocyte electro-mechanical function [1] that predicted a significant role of the intra- and extracellular mechanical factors in arrhythmogenesys. Model prediction was verified in experiments on papillary muscles from the right ventricle of guinea pigs overloaded with calcium [2].

Methods and Algorithms: We utilized the cellular model to study effects of the electromechanical coupling between cardiomyocytes in a 1D heterogeneous muscle strand formed of $90 \%$ of normal (N) cardiomyocytes and $10 \%$ of sub-critical (SC) cardiomyocytes with decreased $\mathrm{Na}^{+}-\mathrm{K}^{+}$pump activity. Single $\mathrm{SC}$-cardiomyocytes did not demonstrate spontaneous activity during isometric contractions at a reference length. Regular fiber twitches at the reference initial cell length were induced by 1 bps electrical stimulation applied at an edge of the strand. Excitation spread along the tissue via electro-diffusional cell coupling followed by cell contractions and force development.

Results: Mechanical interactions between N- and SC-cells in the tissue resulted in the spontaneous activity emerged in the SC-zone between the regular stimuli. If the excitation wave spread from SC- to N-region, the SC-cells developed delayed after-depolarizations (DAD) that caused a slowly developing beat-to-beat decrease in the force of fiber contraction. If the excitation spread in opposite direction, DAD in the SCcells induced reflected downward excitation waves capturing the normal region and followed by extrasystoles in the whole fiber.

Conclusion: The results obtained in the model suggest that ectopic activity may emerge in a sub-critical myocardial region, e.g. comprising cardiomyocytes with moderately depressed $\mathrm{N}+\mathrm{K}+$ pump, due to its mechanical interactions in the myocardial tissue. Moreover, such ectopic zone may expand by capturing normal regions in myocardium via the electro-mechanical coupling between cardiomyocytes.

Acknowledgements: Supported by the RFBR (14-01-00885, 14-01-31134), by Presidium of UB RAS (12M-14-2009, 12-П-4-1067) and by UrFU (Act 211 of RF Government \# 02.A03.21.0006).

\section{References}

1. Katsnelson L.B. et al. Contribution of mechanical factors to arrhythmogenesis in calcium overloaded cardiomyocytes: Model predictions and experiments. Progress Biophysics Mol Biol. 2011;107(1):81-89.

2. Lashin S.A., Matushkin Yu.G. Haploid evolutionary constructor: new features and further challenges. In Silico. Biol. 2012;11(3):125-135. 